\title{
NEW WEAK MARTINGALE HARDY SPACES OF MUSIELAK-ORLICZ TYPE
}

\author{
Anming Yang \\ Fudan University, School of Mathematical Sciences \\ Shanghai 200433, P. R. China; amyang14@fudan.edu.cn
}

\begin{abstract}
The main purpose of this paper is to introduce the weak Musielak-Orlicz martingale spaces and establish several weak atomic decompositions for them. With the help of weak atomic decompositions, a sufficient condition for sublinear operators defined on weak MusielakOrlicz martingale spaces to be bounded is given. Using the sufficient condition, a series of martingale inequalities are obtained. These results are generalizations of the previous results of weak martingale Hardy spaces and weak martingale Orlicz-Hardy spaces.
\end{abstract}

\section{Introduction}

The real-variable theory of Hardy spaces, initiated by Stein and Weiss [21], develops rapidly and plays an important role in various fields of analysis, see, for example, $[2,5,19,20]$. The weak Hardy space was originally introduced by Fefferman and Soria [4], and then undergone a vast research, see, for example, [3, 7, 18]. Meanwhile, as a counterpart to the Hardy spaces of functions, the martingale Hardy spaces and weak martingale Hardy spaces were also studied by many authors, see, for example, $[6,9,16,22,23]$. We know that the atomic decompositions of the Hardy spaces $H^{p}\left(\mathbf{R}^{n}\right)$, which were obtained by Coifman [1] when $n=1$ and Latter [14] when $n>1$, are very important in the real-variable theory. Just as they do in the theory of Hardy spaces of functions, atomic decompositions also play a key role in martingale theory.

As is well known, Weisz [23] gave several atomic decompositions on martingale Hardy spaces and proved many important theorems with the help of atomic decompositions. Since then, atomic decompositions were established for many other martingale spaces, see [9] for weak martingale Hardy spaces, [10, 12] for martingale Hardy-Lorentz spaces, [17] for Orlicz-Hardy martingale spaces, [11] for weak Orlicz-Hardy martingale spaces, [24] for weak Orlicz-Lorentz martingale spaces, [8] for Lorentz-Karamata martingale spaces, and so on.

Recently, Liang, Yang and Jiang [15] introduced the weak Musielak-Orlicz Hardy spaces and the results in [15] generalized the real-variable theory of weak Hardy spaces and weighted weak Hardy spaces. Inspired by [15], we introduce the martingale version of weak Musielak-Orlicz Hardy spaces. The results obtained in this paper will generalize the previous results of weak martingale Hardy spaces and weak martingale Orlicz-Hardy spaces.

The paper is organized as follows. In the next section, some preliminaries and the notion of weak Musielak-Orlicz martingale spaces are introduced. In Section 3, several atomic decompositions of weak Musielak-Orlicz martingale spaces are formulated. In Section 4, using the atomic decompositions formulated in Section 3, a sufficient condition for sublinear operators defined on the weak Musielak-Orlicz

https://doi.org/10.5186/aasfm.2017.4249

2010 Mathematics Subject Classification: Primary 60G46; Secondary 60G42.

Key words: Musielak-Orlicz function, weak Musielak-Orlicz martingale space, atomic decomposition, martingale inequality. 
martingale spaces to be bounded is given. We also obtain a series of martingale inequalities with the help of the sufficient condition.

Throughout this paper, the set of integers and the set of nonnegative integers are always denoted by $\mathbf{Z}$ and $\mathbf{N}$, respectively. We use $C$ to denote (possibly vary from line to line) constants that are independent of the essential variables.

\section{Preliminaries}

Let $(\Omega, \mathcal{F}, \mathbf{P})$ be a complete probability space. Following [13] and [15], we first introduce the notion of Musielak-Orlicz functions. Recall that a function $\Phi:[0, \infty) \rightarrow$ $[0, \infty)$ is called an Orlicz function if it is nondecreasing, $\Phi(0)=0, \Phi(t)>0$ for all $t \in(0, \infty)$ and $\lim _{t \rightarrow \infty} \Phi(t)=\infty$. The function $\Phi$ is said to be of upper (resp. lower) type $p$ for certain $p \in[0, \infty)$ if there exists a positive constant $C_{\Phi}$ such that, for all $s \in[1, \infty)$ (resp. $s \in[0,1]$ ) and $t \in[0, \infty), \Phi(s t) \leq C_{\Phi} s^{p} \Phi(t)$.

A function $\varphi: \Omega \times[0, \infty) \rightarrow[0, \infty)$ is called a Musielak-Orlicz function if the function $\varphi(x, \cdot):[0, \infty) \rightarrow[0, \infty)$ is an Orlicz function for any $x \in \Omega$ and the function $\varphi(\cdot, t): \Omega \rightarrow[0, \infty)$ is a measurable function for each $t \in[0, \infty)$. A typical example of Musielak-Orlicz functions is $\varphi(x, t)=f(x) g(t)$, where $f$ is a positive measurable function on $\Omega$ and $g$ is an Orlicz function on $[0, \infty)$. Of course, there exist MusielakOrlicz functions which are not of that form. An example is $\varphi(x, t)=\frac{t}{f(x)+\log (e+t)}$, where $f$ is a positive measurable function on $\Omega$.

Let $\varphi$ be a Musielak-Orlicz function. $\varphi$ is said to be of uniformly upper (resp. lower) type $p$ for certain $p \in[0, \infty)$ if there exists a positive constant $C_{\varphi}$ such that, for all $x \in \Omega, s \in\left[1, \infty\right.$ ) (resp. $s \in[0,1]$ ) and $t \in[0, \infty), \varphi(x, s t) \leq C_{\varphi} s^{p} \varphi(x, t)$. Without loss of generality, we can assume that $C_{\varphi} \geq 1$. Throughout the whole paper, we always assume that $\varphi$ satisfies the following assumption.

Assumption 2.1. $\varphi: \Omega \times[0, \infty) \rightarrow[0, \infty)$ is a Musielak-Orlicz function and $\varphi$ is of uniformly lower type $\bar{p}$ for certain $\bar{p} \in(0,1]$ and of uniformly upper type 1 .

In what follows, for any measurable subset $E$ of $\Omega$ and $t \in[0, \infty)$, we denote $\int_{E} \varphi(x, t) d \mathbf{P}$ simply by $\varphi(E, t)$. We now list some properties of the Musielak-Orlicz functions which satisfy Assumption 2.1 in the following proposition. These properties will be frequently used in the sequel and the proofs for them are evident.

Proposition 2.2. Let $\varphi$ satisfy Assumption 2.1, $E$ and $F$ be measurable subsets of $\Omega, x \in \Omega$ and $s, t \in[0, \infty)$, we have

(i) if $s \leq t$, then $\varphi(x, s) \leq \varphi(x, t), \varphi(E, s) \leq \varphi(E, t)$;

(ii) if $E \subset F$, then $\varphi(E, t) \leq \varphi(F, t)$;

(iii) $\varphi(E \cup F, t) \leq \varphi(E, t)+\varphi(F, t)$;

(iv) if $s \in[0,1]$, then $\varphi(E, s t) \leq C_{\varphi} s^{\bar{p}} \varphi(E, t)$;

(v) if $s \in[1, \infty)$, then $\varphi(E, s t) \leq C_{\varphi} s \varphi(E, t)$.

Let $\varphi$ satisfy Assumption 2.1, the weak Musielak-Orlicz space $w L_{\varphi}(\Omega, \mathcal{F}, \mathbf{P})$ is defined as the space of all measurable functions $f$ such that $\|f\|_{w L_{\varphi}}<\infty$, where

$$
\|f\|_{w L_{\varphi}}=\inf \left\{c>0: \sup _{\alpha>0} \varphi\left(\{x \in \Omega:|f(x)|>\alpha\}, \frac{\alpha}{c}\right) \leq 1\right\} .
$$

We now introduce weak Musielak-Orlicz martingale spaces. Let $(\Omega, \mathcal{F}, \mathbf{P})$ be the above complete probability space and $\left\{\mathcal{F}_{n}\right\}_{n \geq 0}$ be a nondecreasing sequence of sub- $\sigma$-algebras of $\mathcal{F}$ such that $\mathcal{F}=\sigma\left(\bigcup_{n \geq 0} \mathcal{F}_{n}\right)$. The expectation operator and the 
conditional expectation operators related to $\mathcal{F}_{n}$ are denoted by $\mathbf{E}$ and $\mathbf{E}_{n}$, respectively. Denote by $\mathcal{M}$ the set of all martingales $f=\left(f_{n}\right)_{n \geq 0}$ relative to $\left\{\mathcal{F}_{n}\right\}_{n \geq 0}$ such that $f_{0}=0$. For $f \in \mathcal{M}$, denote its martingale difference by $d_{n} f=f_{n}-f_{n-1}(n \geq 0$, with convention $f_{-1}=0$ ). Then the maximal function, the quadratic variation and the conditional quadratic variation of a martingale $f$ are defined by

$$
\begin{aligned}
& f_{n}^{*}=\sup _{0 \leq i \leq n}\left|f_{i}\right|, \quad f^{*}=\sup _{i \geq 0}\left|f_{i}\right| ; \quad S_{n}(f)=\left(\sum_{i=1}^{n}\left|d_{i} f\right|^{2}\right)^{\frac{1}{2}}, \quad S(f)=\left(\sum_{i=1}^{\infty}\left|d_{i} f\right|^{2}\right)^{\frac{1}{2}} ; \\
& s_{n}(f)=\left(\sum_{i=1}^{n} \mathbf{E}_{i-1}\left|d_{i} f\right|^{2}\right)^{\frac{1}{2}}, \quad s(f)=\left(\sum_{i=1}^{\infty} \mathbf{E}_{i-1}\left|d_{i} f\right|^{2}\right)^{\frac{1}{2}} .
\end{aligned}
$$

Let $\Lambda$ be the collection of all sequences $\left(\lambda_{n}\right)_{n \geq 0}$ of nondecreasing, nonnegative and adapted functions, set $\lambda_{\infty}=\lim _{n \rightarrow \infty} \lambda_{n}$. For $\varphi$ satisfies Assumption 2.1 and $f \in \mathcal{M}$, let

$$
\begin{aligned}
& \Lambda\left[w Q_{\varphi}\right](f)=\left\{\left(\lambda_{n}\right)_{n \geq 0} \in \Lambda: S_{n}(f) \leq \lambda_{n-1}(n \geq 1), \lambda_{\infty} \in w L_{\varphi}\right\} \\
& \Lambda\left[w D_{\varphi}\right](f)=\left\{\left(\lambda_{n}\right)_{n \geq 0} \in \Lambda:\left|f_{n}\right| \leq \lambda_{n-1}(n \geq 1), \lambda_{\infty} \in w L_{\varphi}\right\} .
\end{aligned}
$$

The weak Musielak-Orlicz martingale spaces are defined as follows,

$$
\begin{aligned}
& w H_{\varphi}^{*}=\left\{f \in \mathcal{M}:\|f\|_{w H_{\varphi}^{*}}=\left\|f^{*}\right\|_{w L_{\varphi}}<\infty\right\}, \\
& w H_{\varphi}^{S}=\left\{f \in \mathcal{M}:\|f\|_{w H_{\varphi}^{S}}=\|S(f)\|_{w L_{\varphi}}<\infty\right\}, \\
& w H_{\varphi}^{s}=\left\{f \in \mathcal{M}:\|f\|_{w H_{\varphi}^{s}}=\|s(f)\|_{w L_{\varphi}}<\infty\right\}, \\
& w Q_{\varphi}=\left\{f \in \mathcal{M}:\|f\|_{w Q_{\varphi}}=\inf _{\left(\lambda_{n}\right)_{n \geq 0} \in \Lambda\left[w Q_{\varphi}\right](f)}\left\|\lambda_{\infty}\right\|_{w L_{\varphi}}<\infty\right\}, \\
& w D_{\varphi}=\left\{f \in \mathcal{M}:\|f\|_{w D_{\varphi}}=\inf _{\left(\lambda_{n}\right)_{n \geq 0} \in \Lambda\left[w D_{\varphi}\right](f)}\left\|\lambda_{\infty}\right\|_{w L_{\varphi}}<\infty\right\} .
\end{aligned}
$$

If taking $\varphi(x, t)=t^{p}$ or $\Phi(t)$ (where $\Phi(t)$ is an Orlicz function), then we get the usual weak martingale Hardy spaces and weak martingale Orlicz-Hardy spaces, respectively.

We conclude this section by introducing the notion of atoms.

Definition 2.3. A measurable function $a$ is said to be a weak atom of the first category (or of the second category, of the third category, respectively) if there exists a stopping time $\nu(\nu$ is called the stopping time associated with $a$ ) such that

(i) $a_{n}=\mathbf{E}_{n}(a)=0$, (if $\nu \geq n$ ),

(ii) $\|s(a)\|_{\infty}<\infty$ (or (ii') $\|S(a)\|_{\infty}<\infty$, (ii') $\left\|a^{*}\right\|_{\infty}<\infty$, respectively).

These three categroy weak atoms are briefly called w-1-atom, w-2-atom and w3-atom, respectively.

\section{Atomic decompositions}

We now establish the atomic decompositions of the weak Musielak-Orlicz martingale spaces.

Theorem 3.1. Let $\varphi$ satisfy Assumption 2.1, $f=\left(f_{n}\right)_{n \geq 0} \in w H_{\varphi}^{s}$, then there exist a sequence $\left\{a^{k}\right\}_{k \in \mathbf{Z}}$ of $w$-1-atoms and the corresponding stopping times $\left\{\nu_{k}\right\}_{k \in \mathbf{Z}}$ such that

(i) $f_{n}=\sum_{k \in \mathbf{Z}} a_{n}^{k},(n \in \mathbf{N})$, 
(ii) $s\left(a^{k}\right) \leq A \cdot 2^{k}$ for some constant $A>0$ and

$$
\inf \left\{c>0: \sup _{k \in \mathbf{Z}} \varphi\left(\left\{\nu_{k}<\infty\right\}, \frac{2^{k}}{c}\right) \leq 1\right\}<\infty .
$$

Conversely, if the martingale $f$ has the above decomposition, then $f \in w H_{\varphi}^{s}$ and

$$
\|f\|_{w H_{\varphi}^{s}} \sim \inf \inf \left\{c>0: \sup _{k \in \mathbf{Z}} \varphi\left(\left\{\nu_{k}<\infty\right\}, \frac{2^{k}}{c}\right) \leq 1\right\}
$$

where the first infimum is taken over all the preceding decompositions of $f$.

Proof. Assume that $f \in w H_{\varphi}^{s}$. For each $k \in \mathbf{Z}$, define stopping time as follows,

$$
\nu_{k}=\inf \left\{n \in \mathbf{N}: s_{n+1}(f)>2^{k}\right\}, \quad(\inf \emptyset=\infty) .
$$

Let $f^{\nu_{k}}=\left(f_{n \wedge \nu_{k}}\right)_{n \geq 0}$ be the stopped martingale, then $s\left(f^{\nu_{k}}\right) \leq 2^{k}$ and

$$
f_{n}=\sum_{k \in \mathbf{Z}}\left(f_{n}^{\nu_{k+1}}-f_{n}^{\nu_{k}}\right) .
$$

Let $a_{n}^{k}=f_{n}^{\nu_{k+1}}-f_{n}^{\nu_{k}}$, then for each fixed $k \in \mathbf{Z}, a^{k}=\left\{a_{n}^{k}\right\}_{n \geq 0}$ is a martingale. By the sublinearity of the operator $s$ we have

$$
s\left(a^{k}\right) \leq s\left(f^{\nu_{k+1}}\right)+s\left(f^{\nu_{k}}\right) \leq 3 \cdot 2^{k} .
$$

This means that $\left\{a_{n}^{k}\right\}_{n \geq 0}$ is a $L_{2}$-bounded martingale, then $\left\{a_{n}^{k}\right\}_{n \geq 0}$ converges in $L_{2}$. Denote the limit still by $a^{k}$, then $\mathbf{E}_{n}\left(a^{k}\right)=a_{n}^{k}$. For $\nu_{k} \geq n, a_{n}^{k}=f_{n}^{\nu_{k+1}}-f_{n}^{\nu_{k}}=0$. So $a^{k}$ is really a w-1-atom and (i) holds. To prove (ii), it is easy to see that $\left\{\nu_{k}<\right.$ $\infty\}=\left\{s(f)>2^{k}\right\}$. By the definition of the norm $\|\cdot\|_{w H_{\varphi}^{s}}$ we have

$$
\varphi\left(\left\{\nu_{k}<\infty\right\}, \frac{2^{k}}{\|f\|_{w H_{\varphi}^{s}}}\right)=\varphi\left(\left\{s(f)>2^{k}\right\}, \frac{2^{k}}{\|s(f)\|_{w L_{\varphi}}}\right) \leq 1 .
$$

Hence

$$
\inf \left\{c>0: \sup _{k \in \mathbf{Z}} \varphi\left(\left\{\nu_{k}<\infty\right\}, \frac{2^{k}}{c}\right) \leq 1\right\} \leq\|f\|_{w H_{\varphi}^{s}}<\infty .
$$

Conversely, suppose that there exist a sequence $\left\{a^{k}\right\}_{k \in \mathbf{Z}}$ of $\mathrm{w}$-1-atoms such that (i) and (ii) hold. Let

$$
M=\inf \left\{c>0: \sup _{k \in \mathbf{Z}} \varphi\left(\left\{\nu_{k}<\infty\right\}, \frac{2^{k}}{c}\right) \leq 1\right\} .
$$

For $A$ in (ii), without loss of generality, we assume $A=2^{b}$, where $b$ is a nonnegative integer. For any fixed $\alpha>0$, choose $k_{0} \in \mathbf{Z}$ such that $2^{k_{0}} \leq \alpha<2^{k_{0}+1}$. Let

$$
f=\sum_{k \in \mathbf{Z}} a^{k}=g+h,
$$

where $g=\sum_{k=-\infty}^{k_{0}-1} a^{k}$ and $h=\sum_{k=k_{0}}^{\infty} a^{k}$. From (ii) we have

$$
s(g) \leq \sum_{k=-\infty}^{k_{0}-1} s\left(a^{k}\right) \leq \sum_{k=-\infty}^{k_{0}-1} A \cdot 2^{k}=A \cdot 2^{k_{0}} \leq A \alpha .
$$

By the sublinearity of the operator $s$ we have $s(f) \leq s(g)+s(h)$. Then

$$
\{s(f)>2 A \alpha\} \subset\{s(g)>A \alpha\} \cup\{s(h)>A \alpha\}=\{s(h)>A \alpha\} .
$$


Since $s(h) \leq \sum_{k=k_{0}}^{\infty} s\left(a^{k}\right)$ and $\left\{s\left(a^{k}\right)>0\right\} \subset\left\{\nu_{k}<\infty\right\}$, we have

$$
\{s(f)>2 A \alpha\} \subset\{s(h)>A \alpha\} \subset\{s(h)>0\} \subset \bigcup_{k=k_{0}}^{\infty}\left\{s\left(a^{k}\right)>0\right\} \subset \bigcup_{k=k_{0}}^{\infty}\left\{\nu_{k}<\infty\right\} .
$$

Then

$$
\varphi\left(\{s(f)>2 A \alpha\}, \frac{2 A \alpha}{2 M}\right) \leq \varphi\left(\bigcup_{k=k_{0}}^{\infty}\left\{\nu_{k}<\infty\right\}, \frac{A \alpha}{M}\right) \leq \sum_{k=k_{0}}^{\infty} \varphi\left(\left\{\nu_{k}<\infty\right\}, \frac{A \alpha}{M}\right) .
$$

Since $A=2^{b}$ and $2^{k_{0}} \leq \alpha<2^{k_{0}+1}$, then $2^{k_{0}} \leq 2^{b+k_{0}} \leq A \alpha<2^{b+k_{0}+1}$. Let

$$
\sum_{k=k_{0}}^{\infty} \varphi\left(\left\{\nu_{k}<\infty\right\}, \frac{A \alpha}{M}\right)=G+H
$$

where $G=\sum_{k=k_{0}}^{b+k_{0}} \varphi\left(\left\{\nu_{k}<\infty\right\}, \frac{A \alpha}{M}\right)$ and $H=\sum_{k=b+k_{0}+1}^{\infty} \varphi\left(\left\{\nu_{k}<\infty\right\}, \frac{A \alpha}{M}\right)$. Hence we have $\varphi\left(\{s(f)>2 A \alpha\}, \frac{2 A \alpha}{2 M}\right) \leq G+H$. By the definition of $M$ we know that $\varphi\left(\left\{\nu_{k}<\infty\right\}, \frac{2^{k}}{M}\right) \leq 1,(k \in \mathbf{Z})$.

Since $\varphi$ is of uniformly upper type 1 , we have

$$
\begin{aligned}
G & =\sum_{k=k_{0}}^{b+k_{0}} \varphi\left(\left\{\nu_{k}<\infty\right\}, \frac{A \alpha}{M}\right) \leq \sum_{k=k_{0}}^{b+k_{0}} \varphi\left(\left\{\nu_{k}<\infty\right\}, 2^{b+k_{0}+1-k} \frac{2^{k}}{M}\right) \\
& \leq \sum_{k=k_{0}}^{b+k_{0}} C_{\varphi} 2^{b+k_{0}+1-k} \varphi\left(\left\{\nu_{k}<\infty\right\}, \frac{2^{k}}{M}\right) \leq C_{\varphi} \sum_{k=k_{0}}^{b+k_{0}} 2^{b+k_{0}+1-k}=C_{\varphi}\left(2^{b+2}-2\right)=C_{1} .
\end{aligned}
$$

And since $\varphi$ is also of uniformly lower type $\bar{p}$ for some $\bar{p} \in(0,1]$, then

$$
\begin{aligned}
H & =\sum_{k=b+k_{0}+1}^{\infty} \varphi\left(\left\{\nu_{k}<\infty\right\}, \frac{A \alpha}{M}\right) \leq \sum_{k=b+k_{0}+1}^{\infty} \varphi\left(\left\{\nu_{k}<\infty\right\}, 2^{b+k_{0}+1-k} \frac{2^{k}}{M}\right) \\
& \leq \sum_{k=b+k_{0}+1}^{\infty} C_{\varphi}\left(2^{b+k_{0}+1-k}\right)^{\bar{p}} \varphi\left(\left\{\nu_{k}<\infty\right\}, \frac{2^{k}}{M}\right) \leq C_{\varphi} \sum_{k=b+k_{0}+1}^{\infty} 2^{\bar{p}\left(b+k_{0}+1-k\right)} \\
& =C_{\varphi} \frac{2^{\bar{p}}}{2^{\bar{p}}-1}=C_{2} .
\end{aligned}
$$

Since $C_{1}+C_{2}>1$, then

$$
\begin{aligned}
\varphi\left(\{s(f)>2 A \alpha\}, \frac{2 A \alpha}{\left(C_{\varphi}\left(C_{1}+C_{2}\right)\right)^{\frac{1}{\bar{p}}} 2 M}\right) & \leq C_{\varphi} \frac{1}{C_{\varphi}\left(C_{1}+C_{2}\right)} \varphi\left(\{s(f)>2 A \alpha\}, \frac{2 A \alpha}{2 M}\right) \\
& \leq C_{\varphi} \frac{1}{C_{\varphi}\left(C_{1}+C_{2}\right)}\left(C_{1}+C_{2}\right)=1 .
\end{aligned}
$$

Hence $s(f) \in w L_{\varphi}$ and $\|s(f)\|_{w L_{\varphi}} \leq 2\left(C_{\varphi}\left(C_{1}+C_{2}\right)\right)^{\frac{1}{\bar{p}}} M$. Consequently, $f \in w H_{\varphi}^{s}$ and

$$
\|f\|_{w H_{\varphi}^{s}} \sim \inf \inf \left\{c>0: \sup _{k \in \mathbf{Z}} \varphi\left(\left\{\nu_{k}<\infty\right\}, \frac{2^{k}}{c}\right) \leq 1\right\}
$$

where the first infimum is taken over all the preceding decompositions of $f$. The proof of the theorem is complete. 
Theorem 3.2. Let $\varphi$ satisfy Assumption 2.1, $f=\left(f_{n}\right)_{n \geq 0} \in w Q_{\varphi}$. Then there exist a sequence $\left\{a^{k}\right\}_{k \in \mathbf{Z}}$ of $w_{\text {-2-atoms }}$ and the corresponding stopping times $\left\{\nu_{k}\right\}_{k \in \mathbf{Z}}$ such that

(i) $f_{n}=\sum_{k \in \mathbf{Z}} a_{n}^{k},(n \in \mathbf{N})$,

(ii) $S\left(a^{k}\right) \leq A \cdot 2^{k}$ for some constant $A>0$ and

$$
\inf \left\{c>0: \sup _{k \in \mathbf{Z}} \varphi\left(\left\{\nu_{k}<\infty\right\}, \frac{2^{k}}{c}\right) \leq 1\right\}<\infty .
$$

Conversely, if the martingale $f$ has the above decomposition, then $f \in w Q_{\varphi}$ and

$$
\|f\|_{w Q_{\varphi}} \sim \inf \inf \left\{c>0: \sup _{k \in \mathbf{Z}} \varphi\left(\left\{\nu_{k}<\infty\right\}, \frac{2^{k}}{c}\right) \leq 1\right\}
$$

where the first infimum is taken over all the preceding decompositions of $f$.

Proof. Let $f \in w Q_{\varphi}$, for each $k \in \mathbf{Z}$, the stopping time are defined in this case by

$$
\nu_{k}=\inf \left\{n \in \mathbf{N}: \lambda_{n}>2^{k}\right\}, \quad(\inf \emptyset=\infty),
$$

where $\left\{\lambda_{n}\right\}_{n \geq 0}$ is the sequence in the definition of $w Q_{\varphi}$.

Let $a_{n}^{k}=f_{n}^{\nu_{k+1}}-f_{n}^{\nu_{k}}$, then $f_{n}=\sum_{k \in \mathbf{Z}} a_{n}^{k}$. Since $S\left(f^{\nu_{k}}\right)=S_{\nu_{k}}(f) \leq \lambda_{\nu_{k-1}} \leq 2^{k}$, then by the sublinearity of the operator $S$ we have $S\left(a^{k}\right) \leq S\left(f^{\nu_{k+1}}\right)+S\left(f^{\nu_{k}}\right) \leq 3 \cdot 2^{k}$. This means $\left\{a_{n}^{k}\right\}_{n \geq 0}$ is $L_{2}$-bounded and converges in $L_{2}$. Denote the limit still by $a^{k}$, then $\mathbf{E}_{n}\left(a^{k}\right)=a_{n}^{k}$. For $\nu_{k} \geq n, a_{n}^{k}=f_{n}^{\nu_{k+1}}-f_{n}^{\nu_{k}}=0$. So $a^{k}$ is really a w-2-atom and (i) holds. To prove (ii), since $\left\{\nu_{k}<\infty\right\}=\left\{\lambda_{\infty}>2^{k}\right\}$, then

$$
\varphi\left(\left\{\nu_{k}<\infty\right\}, \frac{2^{k}}{\left\|\lambda_{\infty}\right\|_{w L_{\varphi}}}\right)=\varphi\left(\left\{\lambda_{\infty}>2^{k}\right\}, \frac{2^{k}}{\left\|\lambda_{\infty}\right\|_{w L_{\varphi}}}\right) \leq 1
$$

Then by the definition of the norm $\|\cdot\|_{w Q_{\varphi}}$ we have

$$
\inf \left\{c>0: \sup _{k \in \mathbf{Z}} \varphi\left(\left\{\nu_{k}<\infty\right\}, \frac{2^{k}}{c}\right) \leq 1\right\} \leq\left\|\lambda_{\infty}\right\|_{w L_{\varphi}}<\infty .
$$

Conversely, suppose that there exist a sequence $\left\{a^{k}\right\}_{k \in \mathbf{Z}}$ of w-2-atoms such that (i) and (ii) hold. Let

$$
M=\inf \left\{c>0: \sup _{k \in \mathbf{Z}} \varphi\left(\left\{\nu_{k}<\infty\right\}, \frac{2^{k}}{c}\right) \leq 1\right\} .
$$

Define $\lambda_{n}=\sum_{k \in \mathbf{Z}} \chi_{\left\{\nu_{k} \leq n\right\}}\left\|S\left(a^{k}\right)\right\|_{\infty}$. Then $\left(\lambda_{n}\right)_{n \geq 0}$ is a nondecreasing, nonnegative and adapted sequence. Since $\left\{\nu_{k}>n\right\} \subset\left\{S_{n+1}\left(a^{k}\right)=0\right\}$, we have

$$
S_{n+1}(f) \leq \sum_{k \in \mathbf{Z}} S_{n+1}\left(a^{k}\right)=\sum_{k \in \mathbf{Z}} \chi_{\left\{\nu_{k} \leq n\right\}} S_{n+1}\left(a^{k}\right) \leq \sum_{k \in \mathbf{Z}} \chi_{\left\{\nu_{k} \leq n\right\}}\left\|S\left(a^{k}\right)\right\|_{\infty}=\lambda_{n} .
$$

For any fixed $\alpha>0$, choose $k_{0} \in \mathbf{Z}$ such that $2^{k_{0}} \leq \alpha<2^{k_{0}+1}$. Let

$$
\lambda_{\infty}=\sum_{k \in \mathbf{Z}} \chi_{\left\{\nu_{k}<\infty\right\}}\left\|S\left(a^{k}\right)\right\|_{\infty}=\lambda_{\infty}^{(1)}+\lambda_{\infty}^{(2)},
$$

where $\lambda_{\infty}^{(1)}=\sum_{k=-\infty}^{k_{0}-1} \chi_{\left\{\nu_{k}<\infty\right\}}\left\|S\left(a^{k}\right)\right\|_{\infty}$ and $\lambda_{\infty}^{(2)}=\sum_{k=k_{0}}^{\infty} \chi_{\left\{\nu_{k}<\infty\right\}}\left\|S\left(a^{k}\right)\right\|_{\infty}$.

Replacing $s(g)$ and $s(h)$ in the proof of Theorem 3.1 by $\lambda_{\infty}^{(1)}$ and $\lambda_{\infty}^{(2)}$, respectively. Then we obtain $\|f\|_{w Q_{\varphi}} \leq C M$. The proof of the theorem in complete. 
Theorem 3.3. Let $\varphi$ satisfy Assumption 2.1, $f=\left(f_{n}\right)_{n \geq 0} \in w D_{\varphi}$, then there exist a sequence $\left\{a^{k}\right\}_{k \in \mathbf{Z}}$ of ${ }_{W}$-3-atoms and the corresponding stopping times $\left\{\nu_{k}\right\}_{k \in \mathbf{Z}}$ such that

(i) $f_{n}=\sum_{k \in \mathbf{Z}} a_{n}^{k},(n \in \mathbf{N})$,

(ii) $\left(a^{k}\right)^{*} \leq A \cdot 2^{k}$ for some constant $A>0$ and

$$
\inf \left\{c>0: \sup _{k \in \mathbf{Z}} \varphi\left(\left\{\nu_{k}<\infty\right\}, \frac{2^{k}}{c}\right) \leq 1\right\}<\infty .
$$

Conversely, if the martingale $f$ has the above decomposition, then $f \in w D_{\varphi}$ and

$$
\|f\|_{w D_{\varphi}} \sim \inf \inf \left\{c>0: \sup _{k \in \mathbf{Z}} \varphi\left(\left\{\nu_{k}<\infty\right\}, \frac{2^{k}}{c}\right) \leq 1\right\},
$$

where the first infimum is taken over all the preceding decompositions of $f$.

Proof. We can obtain the desired results easily by replacing w-2-atoms and $S\left(a^{k}\right)$ in the proof of Theorem 3.2 by w-3-atoms and $\left(a^{k}\right)^{*}$, respectively. The proof is complete.

\section{Boundedness of operators}

Now we give an application of the atomic decompositions. A sufficient condition for sublinear operators from weak Musielak-Orlicz martingale spaces to weak Musielak-Orlicz function spaces to be bounded will be given in this section. Using this sufficient condition, we deduce a series of martingale inequalities.

An operator $T: X \rightarrow Y$ is called a sublinear operator if it satisfies $|T(f+g)| \leq$ $|T f|+|T g|$ and $|T(\lambda f)|=|\lambda||T f|$, where $X$ is a martingale spaces and $Y$ is a measurable function space. Before we state the theorems, we need the following assumption.

Assumption 4.1. Suppose that $\varphi$ satisfies Assumption 2.1 and there exist two positive constants $B$ and $D$ such that

$$
B \varphi(y, t) \mathbf{P}(E) \leq \varphi(E, t) \leq D \varphi(y, t) \mathbf{P}(E),
$$

where $t \in[0, \infty), y \in \Omega$ and $E$ is an arbitrary measurable subset of $\Omega$.

For instance, if $\varphi(x, t)=f(x) g(t)$, where $f$ is a positive, bounded and measurable function on $\Omega, g$ is an Orlicz function on $[0, \infty)$ which satisfies Assumption 2.1, then $\varphi(x, t))$ satisfies Assumption 4.1 .

Theorem 4.2. Suppose that $\varphi$ satisfies Assumption 4.1 and $T: L_{2}(\Omega) \rightarrow L_{2}(\Omega)$ is a bounded sublinear operator. If $\mathbf{P}(|T a|>0) \leq C \mathbf{P}(\nu<\infty)$ holds for all ${ }_{\mathrm{w}-1 \text { - }}$ atoms, where $\nu$ is the stopping time associated with $a$. Then there exists a positive constant $C^{\prime}$ such that

$$
\|T f\|_{w L_{\varphi}} \leq C^{\prime}\|f\|_{w H_{\varphi}^{s}}, \quad\left(f \in w H_{\varphi}^{s}\right) .
$$

Proof. Assume that $f \in w H_{\varphi}^{s}$. By Theorem 3.1 we know that $f$ can be decomposed into the sum of a sequence of w-1-atoms. For any fixed $\alpha>0$, choose $k_{0} \in \mathbf{Z}$ such that $2^{k_{0}-1} \leq \alpha<2^{k_{0}}$. Let

$$
f=\sum_{k \in \mathbf{Z}} a^{k}=g+h
$$

where $g=\sum_{k=-\infty}^{k_{0}-1} a^{k}$ and $h=\sum_{k=k_{0}}^{\infty} a^{k}$. 
By the sublinearity of the operator $T$ we have $|T f| \leq|T g|+|T h|$, then

$$
\{|T f|>2 \alpha\} \subset\{|T g|>\alpha\} \cup\{|T h|>\alpha\} .
$$

Thus

$$
\varphi\left(\{|T f|>2 \alpha\}, \frac{2 \alpha}{2\|f\|_{w H_{\varphi}^{s}}}\right) \leq \varphi\left(\{|T g|>\alpha\} \cup\{|T h|>\alpha\}, \frac{\alpha}{\|f\|_{w H_{\varphi}^{s}}}\right) \leq I_{1}+I_{2},
$$

where $I_{1}=\varphi\left(\{|T g|>\alpha\}, \frac{\alpha}{\|f\|_{w H_{\varphi}^{s}}}\right)$ and $I_{2}=\varphi\left(\{|T h|>\alpha\}, \frac{\alpha}{\|f\|_{w H_{\varphi}^{s}}}\right)$.

By Theorem 2.11 in [23] we know that for each $k \in \mathbf{Z}$ there exists a constant $C$ such that $\left\|a^{k}\right\|_{2} \leq C\left\|s\left(a^{k}\right)\right\|_{2}$. Then

$$
\|g\|_{2} \leq \sum_{k=-\infty}^{k_{0}-1}\left\|a^{k}\right\|_{2} \leq C \sum_{k=-\infty}^{k_{0}-1}\left\|s\left(a^{k}\right)\right\|_{2} \leq C \sum_{k=-\infty}^{k_{0}-1} 2^{k} \mathbf{P}\left(\nu_{k}<\infty\right)^{\frac{1}{2}}
$$

Let $\nu_{k}$ be the stopping time associated with the weak atom $a^{k}$ in the proof of Theorem 3.1, then for each $k \in \mathbf{Z}$ we have

$$
\varphi\left(\left\{\nu_{k}<\infty\right\}, \frac{2^{k}}{\|f\|_{w H_{\varphi}^{s}}}\right)=\varphi\left(\left\{s(f)>2^{k}\right\}, \frac{2^{k}}{\|f\|_{w H_{\varphi}^{s}}}\right) \leq 1 .
$$

Notice that $\varphi$ satisfies Assumption 4.1, $T$ is bounded from $L_{2}(\Omega)$ to $L_{2}(\Omega)$ and $2^{k_{0}-1} \leq \alpha<2^{k_{0}}$, then

$$
\begin{aligned}
I_{1} & =\varphi\left(\{|T g|>\alpha\}, \frac{\alpha}{\|f\|_{w H_{\varphi}}}\right) \leq D \varphi\left(y, \frac{\alpha}{\|f\|_{w H_{\varphi}}}\right) \mathbf{P}(|T g|>\alpha) \\
& \leq D \varphi\left(y, \frac{\alpha}{\|f\|_{w H_{\varphi}^{s}}}\right) \frac{\|T g\|_{2}^{2}}{\alpha^{2}} \leq C \varphi\left(y, \frac{\alpha}{\|f\|_{w H_{\varphi}}}\right) \frac{\|g\|_{2}^{2}}{\alpha^{2}} \\
& \leq C \varphi\left(y, \frac{\alpha}{\|f\|_{w H_{\varphi}^{s}}}\right)\left(\frac{1}{\alpha} \sum_{k=-\infty}^{k_{0}-1} 2^{k} \mathbf{P}\left(\nu_{k}<\infty\right)^{\frac{1}{2}}\right)^{2} \\
& =C\left(\frac{1}{\alpha} \sum_{k=-\infty}^{k_{0}-1} 2^{k}\left(\varphi\left(y, \frac{\alpha}{\|f\|_{w H_{\varphi}^{s}}}\right) \mathbf{P}\left(\nu_{k}<\infty\right)\right)^{\frac{1}{2}}\right)^{2} \\
& \leq C\left(\frac{1}{\alpha} \sum_{k=-\infty}^{k_{0}-1} 2^{k}\left(\frac{1}{B} \varphi\left(\left\{\nu_{k}<\infty\right\}, \frac{\alpha}{\|f\|_{w H_{\varphi}^{s}}}\right)\right)^{\frac{1}{2}}\right)^{2} \\
& \leq C\left(\frac{1}{\alpha} \sum_{k=-\infty}^{k_{0}-1} 2^{k}\left(\frac{1}{B} C_{\varphi} \frac{\alpha}{2^{k}} \varphi\left(\left\{\nu_{k}<\infty\right\}, \frac{2^{k}}{\|f\|_{w H_{\varphi}^{s}}}\right)\right)^{\frac{1}{2}}\right)^{2} \\
& \leq C\left(\frac{1}{\alpha} \sum_{k=-\infty}^{k_{0}-1} 2^{k}\left(\frac{\alpha}{2^{k}}\right)^{\frac{1}{2}}\right)^{2}=C \frac{2^{k_{0}-1}}{\left(\frac{3}{2}-\sqrt{2}\right) \alpha} \leq \frac{C}{\frac{3}{2}-\sqrt{2}}=C_{1} .
\end{aligned}
$$

On the other hand, by the sublinearity of the operator $T$ we have $|T h| \leq \sum_{k=k_{0}}^{\infty}\left|T a^{k}\right|$, then

$$
\{|T h|>\alpha\} \subset\{|T h|>0\} \subset \bigcup_{k=k_{0}}^{\infty}\left\{\left|T a^{k}\right|>0\right\} .
$$


Then we can obtain

$$
\begin{aligned}
I_{2} & =\varphi\left(\{|T h|>\alpha\}, \frac{\alpha}{\|f\|_{w H_{\varphi}^{s}}}\right) \leq \varphi\left(\bigcup_{k=k_{0}}^{\infty}\left\{\left|T a^{k}\right|>0\right\}, \frac{\alpha}{\|f\|_{w H_{\varphi}^{s}}}\right) \\
& \leq \sum_{k=k_{0}}^{\infty} \varphi\left(\left\{\left|T a^{k}\right|>0\right\}, \frac{\alpha}{\|f\|_{w H_{\varphi}^{s}}}\right) \leq \sum_{k=k_{0}}^{\infty} D \varphi\left(y, \frac{\alpha}{\|f\|_{w H_{\varphi}^{s}}}\right) \mathbf{P}\left(\left|T a^{k}\right|>0\right) \\
& \leq C \sum_{k=k_{0}}^{\infty} \varphi\left(y, \frac{\alpha}{\|f\|_{w H_{\varphi}^{s}}}\right) \mathbf{P}\left(\nu_{k}<\infty\right) \leq C \sum_{k=k_{0}}^{\infty} \frac{1}{B} \varphi\left(\left\{\nu_{k}<\infty\right\}, \frac{\alpha}{\|f\|_{w H_{\varphi}^{s}}}\right) \\
& \leq C \sum_{k=k_{0}}^{\infty} \frac{1}{B} C_{\varphi}\left(\frac{\alpha}{2^{k}}\right)^{\bar{p}} \varphi\left(\left\{\nu_{k}<\infty\right\}, \frac{2^{k}}{\|f\|_{w H_{\varphi}^{s}}}\right) \leq C \sum_{k=k_{0}}^{\infty}\left(\frac{\alpha}{2^{k}}\right)^{\bar{p}} \\
& =\frac{C}{1-2^{-\bar{p}}}\left(\frac{\alpha}{2^{k_{0}}}\right)^{\bar{p}} \leq \frac{C}{1-2^{-\bar{p}}}=C_{2} .
\end{aligned}
$$

Thus

$$
\varphi\left(\{|T f|>2 \alpha\}, \frac{2 \alpha}{2\|f\|_{w H_{\varphi}^{s}}}\right) \leq C_{1}+C_{2} .
$$

Let $C_{3}=\max \left\{C_{1}+C_{2}, \frac{1}{C_{\varphi}}\right\}$, then

$$
\begin{aligned}
\varphi\left(\{|T(f)|>2 \alpha\}, \frac{2 \alpha}{\left(C_{3} C_{\varphi}\right)^{\frac{1}{\bar{p}}} 2\|f\|_{w H_{\varphi}^{s}}}\right) & \leq C_{\varphi} \frac{1}{C_{3} C_{\varphi}} \varphi\left(\{|T(f)|>2 \alpha\}, \frac{2 \alpha}{2\|f\|_{w H_{\varphi}^{s}}}\right) \\
& \leq C_{\varphi} \frac{1}{C_{3} C_{\varphi}}\left(C_{1}+C_{2}\right) \leq 1 .
\end{aligned}
$$

Hence $T f \in w L_{\varphi}$ and

$$
\|T f\|_{w L_{\varphi}} \leq 2\left(C_{3} C_{\varphi}\right)^{\frac{1}{\bar{p}}}\|f\|_{w H_{\varphi}^{s}} .
$$

The proof of the theorem is complete.

On the lines of the proof of Theorem 4.2, using Theorem 3.2 and Theorem 3.3 instead of Theorem 3.1, respectively. We can obtain the following two theorems.

Theorem 4.3. Suppose that $\varphi$ satisfies Assumption 4.1 and $T: L_{2}(\Omega) \rightarrow L_{2}(\Omega)$ is a bounded sublinear operator. If $\mathbf{P}(|T a|>0) \leq C \mathbf{P}(\nu<\infty)$ holds for all $\mathrm{w}-2$ atoms, where $\nu$ is the stopping time associated with $a$. Then there exists a positive constant $C^{\prime}$ such that

$$
\|T f\|_{w L_{\varphi}} \leq C^{\prime}\|f\|_{w Q_{\varphi}}, \quad\left(f \in w Q_{\varphi}\right) .
$$

Theorem 4.4. Suppose that $\varphi$ satisfies Assumption 4.1 and $T: L_{2}(\Omega) \rightarrow L_{2}(\Omega)$ is a bounded sublinear operator. If $\mathbf{P}(|T a|>0) \leq C \mathbf{P}(\nu<\infty)$ holds for all w-3atoms, where $\nu$ is the stopping time associated with $a$. Then there exists a positive constant $C^{\prime}$ such that

$$
\|T f\|_{w L_{\varphi}} \leq C^{\prime}\|f\|_{w D_{\varphi}}, \quad\left(f \in w D_{\varphi}\right) .
$$

Now we can obtain some inequalities for the weak Musielak-Orlicz martingale spaces.

Theorem 4.5. Suppose that $\varphi$ satisfies Assumption 4.1, then for all martingales $f=\left(f_{n}\right)_{n \geq 0}$ the following martingale inequalities hold,

(i) $\|f\|_{w H_{\varphi}^{*}} \leq C\|f\|_{w H_{\varphi}^{s}},\|f\|_{w H_{\varphi}^{S}} \leq C\|f\|_{w H_{\varphi}^{s}}$; 
(ii) $\|f\|_{w H_{\varphi}^{*}} \leq C\|f\|_{w Q_{\varphi}},\|f\|_{w H_{\varphi}^{S}} \leq C\|f\|_{w Q_{\varphi}},\|f\|_{w H_{\varphi}^{s}} \leq C\|f\|_{w Q_{\varphi}}$;

(iii) $\|f\|_{w H_{\varphi}^{*}} \leq C\|f\|_{w D_{\varphi}},\|f\|_{w H_{\varphi}^{S}} \leq C\|f\|_{w D_{\varphi}},\|f\|_{w H_{\varphi}^{s}} \leq C\|f\|_{w D_{\varphi}}$.

Proof. Taking $T f=f^{*}$ and $T f=S(f)$, respectively. By Theorem 4.2 we obtain (i). To prove (ii) and (iii), taking $T f=f^{*}, T f=S(f)$ and $T(f)=s(f)$, respectively. Then (ii) and (iii) are easily proved by using Theorem 4.3 and Theorem 4.4, respectively. The proof of theorem is complete.

Acknowledgements. The author would like to express his gratitude to the referee for his/her careful reading and useful comments .

\section{References}

[1] Colfman, R. R.: A real variable characterization of $H^{p}$. - Studia Math. 51, 1974, 269-274.

[2] Coifman, R. R., and G. Weiss: Extensions of Hardy spaces and their use in analysis. - Bull. Amer. Math. Soc. 83, 1977, 569-645.

[3] Fefferman, C., N. M. Riviére, and Y. Sagher: Interpolation between $H^{p}$ spaces: the real method. - Trans. Amer. Math. Soc. 191, 1974, 75-81.

[4] Fefferman, R., and F. Soria: The space weak $H^{1}$. - Studia Math. 85, 1986, 1-16.

[5] Fefferman, C., and E. M. Stein: $H^{p}$ spaces of several variables. - Acta Math. 129, 1972, $137-193$.

[6] Garsia, A. M.: Martingale inequalities: Seminar notes on recent progress. - Math. Lecture Notes Series, Benjamin Inc., New York, 1973.

[7] He, D. Q.: Square function characterization of weak Hardy spaces. - J. Fourier Anal. Appl. 20, 2014, 1083-1110.

[8] Ho, K.-P.: Atomic decompositions, dual spaces and interpolations of martingale HardyLorentz-Karamata spaces. - Q. J. Math. 65, 2014, 985-1009.

[9] Hou, Y. L., and Y. B. Ren: Weak martingale Hardy spaces and weak atomic decompositions. - Sci. China Math. 49, 2006, 912-921.

[10] Jiao, Y., L. H. Peng, and P. D. Liu: Atomic decompositions of Lorentz martingale spaces and applications. - J. Funct. Spaces Appl. 7, 2009, 153-166.

[11] JiaO, Y., L. Wu, and L. H. Peng: Weak Orlicz-Hardy martingale spaces. - Internat. J. Math. 26, 2015, 1550062, 1-26.

[12] JiAO, Y., L. WU, A. M. YANG, and R. Yı: The predual and John-Nirenberg inequalities on generalized BMO martingale spaces. - Trans. Amer. Math. Soc. 369, 2017, 537-553.

[13] Ky, L. D.: New Hardy spaces of Musielak-Orlicz type and boundedness of sublinear operators. - Integr. Equ. Oper. Theory 78, 2014, 115-150.

[14] Latter, R. H.: A characterization of $H^{p}\left(\mathbf{R}^{n}\right)$ in terms of atoms. - Studia Math. 62, 1978, 93-101.

[15] Liang, Y. Y., D. C. Yang, and R. J. Jiang: Weak Musielak-Orlicz Hardy spaces and applications. - Math. Nachr. 289, 2016, 634-677.

[16] Long, R. L.: Martingale spaces and inequalities. - Peking Univ. Press, Beijing, 1993.

[17] Miyamoto, T., E. Nakai, and G. Sadasue: Martingale Orlicz-Hardy spaces. - Math. Nachr. $285,2012,670-686$.

[18] Quek, T.S., and D. C. YANG: Calderón-Zygmund-type operators on weighted weak Hardy spaces over $\mathbf{R}^{n}$. - Acta Math. Sin. (Engl. Ser.) 16, 2000, 141-160.

[19] Semmes, S.: A primer on Hardy spaces, and some remarks on a theorem of Evans and Müller. - Commun. Partial Differ. Equ. 19, 1994, 277-319.

[20] Stein, E. M.: Harmonic analysis: Real-variable methods, orthogonality, and oscillatory integrals. - Princeton Univ. Press, Princeton, 1993. 
[21] Stein, E. M., and G. WeIss: On the theory of harmonic functions of several variables. I. The theory of $H^{p}$ spaces. - Acta Math. 103, 1960, 25-62.

[22] Weisz, F.: Martingale Hardy spaces for $0<p \leq 1$. - Probab. Theory Related Fields 84, 1990, $361-376$.

[23] Weisz, F.: Martingale Hardy spaces and their applications in Fourier analysis. - Lecture Notes in Math. 1568, Springer-Verlag, Berlin, 1994.

[24] Zhang, C.Z., M. M. Zhong, and X. Y. Zhang: Atomic decompositons of weak OrliczLorentz martingale spaces. - J. Inequal. Appl. 66, 2014, 1-9.

Received 28 August 2016 • Accepted 20 January 2017 